\title{
Clinical outcomes of arthroscopic rotator cuff repair: a retrospective comparison of double-layer, double-row and suture bridge methods
}

Hironori Kakoi ${ }^{1 *} \mathbb{D}$, Toshihiko Izumi ${ }^{1}$, Yasunari Fujii ${ }^{2}$, Satoshi Nagano ${ }^{1}$, Takao Setoguchi ${ }^{3}$, Yasuhiro Ishidou ${ }^{4}$ and Setsuro Komiya'

\begin{abstract}
Background: The suture-bridge (SB) method has recently become the mainstream means of repairing full-thickness rotator cuff tears. However, in some patients the deep and superficial layers have moved in different directions because of delamination of their rotator cuffs. In such cases, a simple suture (double-layer, double-row [DD] method) is used to repair the superficial and deep layers separately. The purpose of this study was to analyze the clinical outcomes and re-tear rates of the DD and SB methods, with patients selected according to the condition of their torn cuffs.

Methods: We retrospectively registered 74 patients with full-thickness rotator cuff tears that had been repaired arthroscopically, 35 shoulders by the DD and 39 by the SB method. Mean ages were 66.1 years in the DD and 62.9 years in the SB group. We evaluated clinical status before and after surgery (Japanese Orthopedic Association [JOA] scores) and re-tear rate. The Wilcoxon signed-ranks test was used to compare JOA scores and active ROM between before and after surgery in each group. Mann-Whitney's $U$ test was used for comparing JOA scores, active ROM, re-tear rates, size of tear, duration of follow-up, sex, and presence of subscapular muscle repair between the DD and SB groups. A hazard ratio of less than $5 \%$ was considered to denote significance.

Results: JOA scores improved significantly in the DD and SB groups from preoperative means of 63.4 and 63.3 points, respectively, to postoperative means of 91.8 and 92.1 points, respectively. The active flexural ROM improved significantly from means of $110.1^{\circ}$ and $100.0^{\circ}$, respectively, to postoperative means of $142.3^{\circ}$ and $142.7^{\circ}$, respectively; the differences between groups were not significant. Re-tear occurred in 5.9\% of the DD (two of 34 shoulders) and $7.9 \%$ of the SB group (three of 38 shoulders); its incidence did not differ significantly between the two groups.

Conclusions: Both the DD and SB methods achieve satisfactory clinical outcomes that do not differ significantly. Our results suggest that careful selection of operative method on the basis of the delamination pattern in patients undergoing RCT may reduce the re-tear rate after utilizing the SB method.
\end{abstract}

Keywords: Arthroscope, Rotator cuff repair, Double-row method, Suture-bridge method, Delamination

\footnotetext{
* Correspondence: kakoi@m2.kufm.kagoshima-u.ac.jp

${ }^{1}$ Department of Orthopedic Surgery, Graduate School of Medical and Dental

Sciences, Kagoshima University, 8-35-1 Sakuragaoka, Kagoshima City

890-8520, Japan

Full list of author information is available at the end of the article
}

(c) The Author(s). 2018 Open Access This article is distributed under the terms of the Creative Commons Attribution 4.0 International License (http://creativecommons.org/licenses/by/4.0/), which permits unrestricted use, distribution, and reproduction in any medium, provided you give appropriate credit to the original author(s) and the source, provide a link to the Creative Commons license, and indicate if changes were made. The Creative Commons Public Domain Dedication waiver (http://creativecommons.org/publicdomain/zero/1.0/) applies to the data made available in this article, unless otherwise stated. 


\section{Background}

Arthroscopic rotator cuff repair (ARCR) using suture anchors has evolved from a single-row (SR) to a double-row (DR) method, the latter being able to reproduce the foot print of the rotator cuff. However, after use of the DR method, mechanical stress is reportedly concentrated in the medial row, resulting in re-tear of the rotator cuff. In recent years, the suture bridge (SB) method has become the mainstream method for performing ARCR. This method spreads mechanical stress across the stump and increases tendon-bone interface pressure. Although several studies have reported excellent results of ARCR by the SB method, some authors have warned an associated risk of failure around the medial anchor, similar to that reported for the DR method. [1] Cho et al. reported failure of the medial rotator cuff when the medial row of mattress sutures passes through the rotator cuff in the SB method and discussed the possibility of strangulation and relatively quick necrosis of the rotator cuff tendon in the medial row. Therefore, although the mechanical characteristics achieved by the SB method are superior to those of other methods, it may not always be the best choice for full thickness rotator cuff (RCT) tears.

"Delamination" denotes horizontal separation of the tendon substance in a torn rotator cuff. Its incidence was recently shown to be as high as $82.8 \%$ of all RCTs. [2] The directions in which the deep and superficial layers retract may differ in delaminated cuffs. Sugaya et al. demonstrated that the double layer, double-row (DD) method of ARCR better achieves structural integrity of a delaminated cuff because it restores each layer to its original anatomical condition separately, [3] whereas with the SB method sutures are passed through the whole rotator cuff. Therefore, we consider that the SB method is contraindicated in patients with delaminated cuffs and different directions of retraction of the deep and superficial layers. However, no published studies have compared outcomes of the DD and SB methods after allocating patients on the basis of the characteristics of delamination.

The purpose of this study was to analyze the clinical outcomes and re-tear rates of the DD and SB method after basing patient selection on the condition of the torn cuff. Our hypothesis was that optimal selection of procedure on the basis of delamination characteristics would improve clinical outcomes and decrease re-tear rates after both methods of ARCR.

\section{Methods}

\section{Patient selection}

From January 2009 to June 2015, 279 ARCRs were performed in our department. SR, DR (including DD), partial repair, or patch graft methods were selected on the basis of the condition of the torn cuff. The SB method was performed from January 2011. The inclusion criteria for this retrospective study were: (1) full-thickness supraspinatus and/or infraspinatus tear that had been repaired by the DD or SB method of ARCR, and (2) follow-up for at least 12 months. The exclusion criteria included (1) solitary subscapular muscle tear, (2) partial repair method, (3) patch graft method, (4) SR method, and (5) revision surgery. After application of these criteria, 74 shoulders were enrolled in this study (Fig. 1).

Tear size was assessed at surgery according to the Cofield classification [4]. Delamination of the ruptured cuff and the direction of retraction of delaminated layers were also assessed by arthroscopic observation intraoperatively. Mean duration of follow-up was 17.1 months and 15.5 months in the DD and SB groups, respectively. No significant differences in age at surgery, sex, duration of postoperative follow-up, or tear size were identified between the two groups. Significantly more patients had undergone subscapularis repair in the SB than in the DD group. Patient characteristics are summarized in Table 1.

\section{Surgical technique}

Surgery was performed under general anesthesia in the beach chair position.

Firstly, the size of the cuff tear was measured arthroscopically using a probe with a scale. The cuff stump was pulled out to determine whether the remaining cuff could sufficiently cover the greater tubercle. When it was not possible to cover the tubercle, the cuff was released and the foot print advanced $1 \mathrm{~cm}$ to the medial side of the humeral head. For tears considered repairable by the $\mathrm{DD}$ or $\mathrm{SB}$ method without excessive tension, one of these methods was chosen according to the presence of delamination and directions of retraction of the two layers as follows. If there was a mismatch in directions of retraction between the deep and superficial layers of a delaminated cuff, the DD method was utilized, that is, both deep and superficial layers of the rotator cuff were separately repaired using a standardized simple suture configuration. If the cuff had not delaminated or the direction of retraction of the deep layer could easily be aligned with that of the superficial layer of a delaminated cuff, the SB method was utilized. If it was considered impossible to repair the RCT without excessive tension by either the DD or SB method, either the SR, partial repair, or patch graft method was utilized.

\section{Postoperative management}

An abduction pillow was used in all cases for 4-6 weeks after surgery depending on the tension of the repaired cuff. Passive range of motion (ROM) exercise was started three weeks postoperatively and active ROM exercises from 6 to 8 weeks postoperatively depending on the tension of the repaired cuff. 


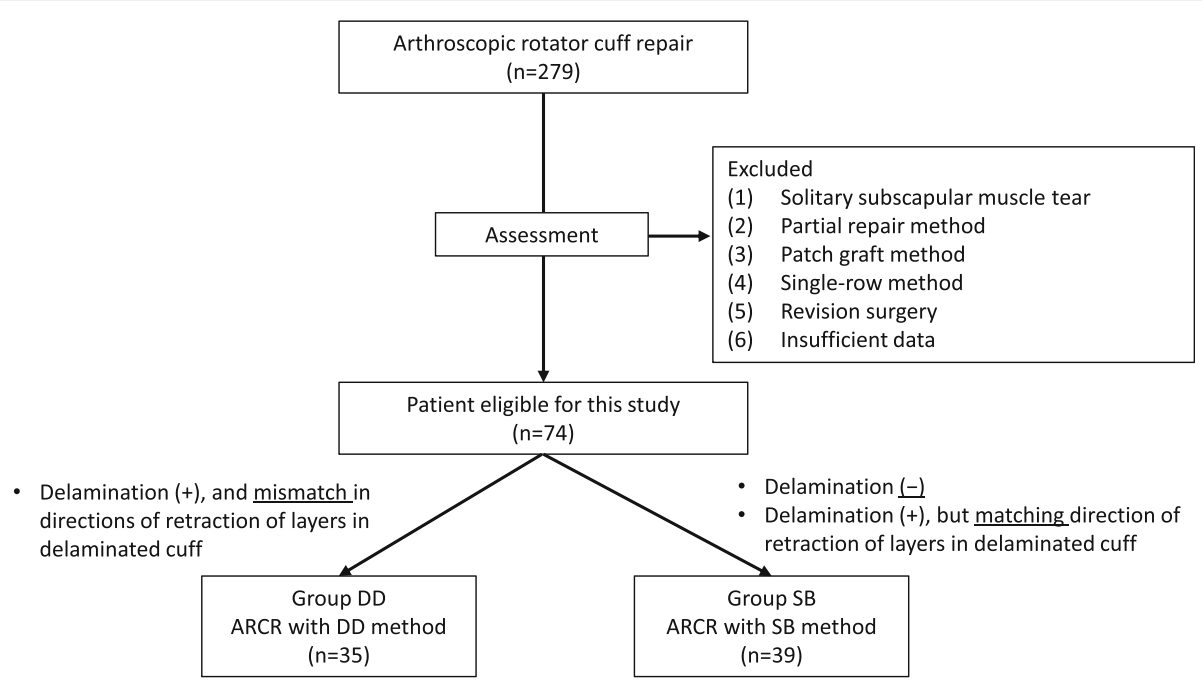

Fig. 1 Flowchart showing study protocol

\section{Clinical assessment}

Clinical outcomes were evaluated preoperatively and at the last follow-up visit using Japanese Orthopedic Association (JOA) scores (Fig. 2) [5]. Active ROM (anterior elevation, external rotation, internal rotation) was also measured preoperatively and at the last follow-up visit. The extent of internal rotation was assessed at the height of the thoracic spinous process reached by a thumb. In accordance with the Ide et al. proposal, the

Table 1 Patient data

\begin{tabular}{llll}
\hline & $\begin{array}{l}\text { Group DD } \\
(n=35)\end{array}$ & $\begin{array}{l}\text { Group SB } \\
(n=39)\end{array}$ & $p$ value \\
Age (y/o) & $(55-7 \pm 6.0$ & $\begin{array}{l}62.9 \pm 1.4 \\
(44-78)\end{array}$ & 0.1718 \\
Sex & 26 & 28 & 0.8109 \\
$\quad$ Male & 9 & 11 & \\
Female & $17.1 \pm 6.6$ & $15.5 \pm 5.9$ & 0.2553 \\
Follow-up period (months) & $(12-36)$ & $(12-37)$ & \\
Repair of subscapularis & 22 & 33 & 0.0336 \\
Tear size & & & \\
Small & 2 & 3 & 0.4473 \\
Medium & 16 & 19 & \\
Large & 12 & 15 & \\
Massive & 5 & 2 & \\
Delamination & & & \\
No & 0 & 13 & \\
Yes & 35 & 26 & \\
Retraction direction of the layers & & & \\
Matched & 35 & & \\
Mismatched & & & \\
\hline
\end{tabular}

first to fifth lumbar vertebrae were described as 13 to 17 , the sacrum as 18 , and the buttocks as 19 [6].

\section{MRI evaluation}

The state of repair of the cuffs was assessed using MRI. MRI evaluation was available at the last follow up for 34 shoulders in the DD and 38 shoulders in the SB group. Re-tears were identified as MRI type 4 or 5 in accordance with the Sugaya classification [7].

\section{Statistical analysis}

The Wilcoxon signed-ranks test was used for statistical analysis of JOA scores and active ROM between before and after surgery in each group. Mann-Whitney's $U$ test was used for comparing JOA scores, active ROM, re-tear rates, size of tear, duration of follow-up, sex, and presence of subscapular muscle repair between the DD and SB groups. A hazard ratio of less than $5 \%$ was considered to denote significance.

\section{Results}

Total JOA score (maximum 100 points) improved significantly from a mean of 63.4 points preoperatively to a mean of 91.8 points at the final follow-up in the DD group. The SB group also showed significant improvement in JOA scores to 92.1 points from a mean of 63.3 points preoperatively. JOA scores did not differ significantly between the two groups, either before surgery or at final follow-up. Analysis of each factor in the JOA scores revealed significant improvements in pain (maximum 30 points), function (maximum 20 points), and ROM (maximum 30 points) in both groups. There were no significant differences between the two groups before surgery or at final follow-up for any JOA score factor (Table 2). 


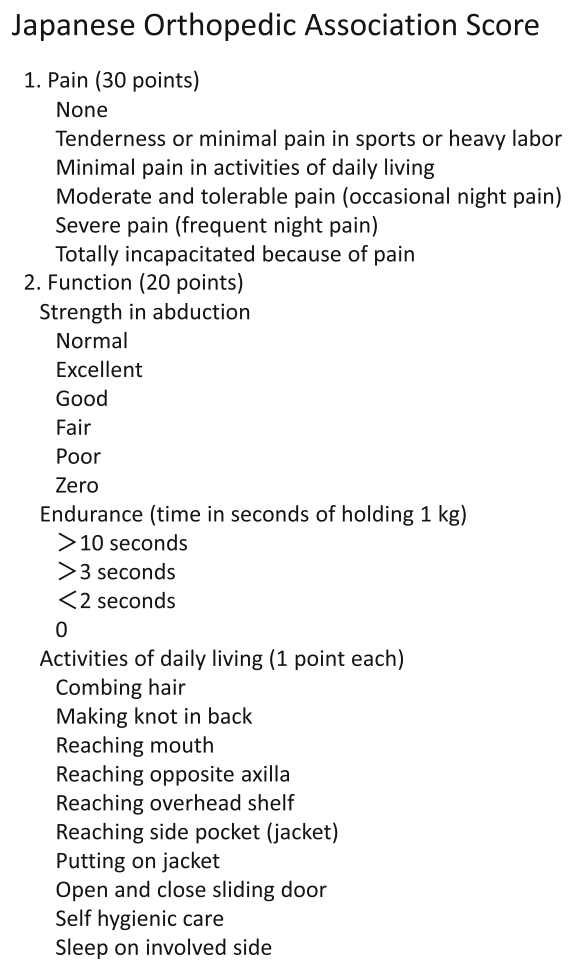

\begin{tabular}{|c|c|}
\hline \multicolumn{2}{|c|}{ 3. Range of motion ( 30 points) } \\
\hline \multicolumn{2}{|l|}{ Elevation } \\
\hline$>150^{\circ}$ & 15 \\
\hline$>120^{\circ}$ & 12 \\
\hline$>90^{\circ}$ & 9 \\
\hline$>60^{\circ}$ & 6 \\
\hline$>30^{\circ}$ & 3 \\
\hline $0^{\circ}$ & 0 \\
\hline \multicolumn{2}{|l|}{ External rotation } \\
\hline$>60^{\circ}$ & 9 \\
\hline$>30^{\circ}$ & 6 \\
\hline$>0^{\circ}$ & 3 \\
\hline$>-20^{\circ}$ & 1 \\
\hline$<-20^{\circ}$ & 0 \\
\hline \multicolumn{2}{|l|}{ Internal rotation } \\
\hline Above Th12 spinous & 6 \\
\hline Above $L 5$ spinous $p$ & 4 \\
\hline Gluteal & 2 \\
\hline Below gluteal & 0 \\
\hline \multicolumn{2}{|c|}{ 4. Radiographic evaluation (5 points) } \\
\hline Normal & 5 \\
\hline Moderate changes & 3 \\
\hline Advanced changes & 0 \\
\hline \multicolumn{2}{|c|}{ 5. Joint stability ( 15 points) } \\
\hline Normal & 15 \\
\hline Slight instability & 10 \\
\hline Severe instability & 5 \\
\hline Relevant history & 0 \\
\hline
\end{tabular}

Fig. 2 Japanese Orthopedic association scoring. This score comprises five items: pain, function, range of motion, imaging findings, and instability. A perfect score is 100 points

Active ROM (anterior elevation, external and internal rotation) was significantly improved in both groups, except for external rotation in the DD group. No significant differences in ROM were found between the two groups preoperatively or at final follow-up, except internal rotation at final follow-up. (Table 3).

Re-tear occurred in $5.9 \%$ of the DD group (two of 34 shoulders) and $7.9 \%$ of the SB group (three of 38 shoulders); its incidence did not differ significantly between the two groups $(p=0.739)$.

\section{Discussion}

The initial fixation force achieved by the SB method is reportedly superior to that achieved by other repair methods using suture anchors because the SB method creates a larger contact area and higher pressure in the

Table 2 Comparison of clinical results

\begin{tabular}{|c|c|c|c|c|c|}
\hline & \multicolumn{2}{|l|}{ Preoperative } & \multicolumn{2}{|l|}{ Final follow-up } & \multirow[t]{2}{*}{$P$} \\
\hline & Mean \pm SD (range) & $P$ value & Mean \pm SD (range) & $P$ value & \\
\hline \multicolumn{6}{|c|}{ JOA score total (Max. 100) } \\
\hline Group DD & $63.4 \pm 8.4(44-78)$ & 0.9654 & $91.8 \pm 7.2(64-100)$ & 0.9393 & $<0.0001$ \\
\hline Group SB & $63.3 \pm 9.2(48-77)$ & & $92.1 \pm 6.2(73-100)$ & & $<0.0001$ \\
\hline \multicolumn{6}{|l|}{ Pain (Max. 30) } \\
\hline Group DD & $9.9 \pm 3.5(5-20)$ & 0.9903 & $27.4 \pm 3.3(20-30)$ & 0.4631 & $<0.0001$ \\
\hline Group SB & $9.9 \pm 3.7(5-20)$ & & $26.7 \pm 4.2(15-30)$ & & $<0.0001$ \\
\hline \multicolumn{6}{|c|}{ Function (Max. 20) } \\
\hline Group DD & $14.2 \pm 3.7(6-19)$ & 0.9957 & $19.1 \pm 2.3(11-20)$ & 0.8389 & $<0.0001$ \\
\hline Group SB & $14.0 \pm 4.3(5-20)$ & & $19.4 \pm 1.1(15-20)$ & & $<0.0001$ \\
\hline \multicolumn{6}{|c|}{ Range of motion (Max.30) } \\
\hline Group DD & $19.6 \pm 5.8(3-30)$ & 0.7858 & $25.0 \pm 4.1(17-30)$ & 0.4733 & 0.0001 \\
\hline Group SB & $20.1 \pm 5.1(6-30)$ & & $26.1 \pm 2.7(22-30)$ & & $<0.0001$ \\
\hline
\end{tabular}

There were no significant differences in JOA scores between the two groups, either before surgery or at final follow-up 
Table 3 Comparison of active range of motion

\begin{tabular}{|c|c|c|c|c|c|}
\hline & \multicolumn{2}{|l|}{ Preoperative } & \multicolumn{2}{|l|}{ final follow-up } & \multirow[t]{2}{*}{$P$ value } \\
\hline & Mean \pm SD(range) & $P$ value & Mean \pm SD(range) & $P$ value & \\
\hline \multicolumn{6}{|c|}{ Anterior elevation $\left({ }^{\circ}\right)$} \\
\hline Group DD & $110.1 \pm 40.7(30-160)$ & 0.1458 & $142.3 \pm 15.17(100-180)$ & 0.9520 & 0.0001 \\
\hline Group SB & $100.0 \pm 36.5(20-150)$ & & $142.7 \pm 15.1(95-175)$ & & $<0.0001$ \\
\hline \multicolumn{6}{|c|}{ External rotation $\left(^{\circ}\right)$} \\
\hline Group DD & $38.3 \pm 23.0(-20-75)$ & 0.2152 & $44.9 \pm 21.5(-5-80)$ & 0.1395 & 0.0817 \\
\hline Group SB & $45.4 \pm 22.0(0-80)$ & & $53.1 \pm 16.3(10-80)$ & & 0.0034 \\
\hline \multicolumn{6}{|c|}{ Internal rotation } \\
\hline Group DD & $14.2 \pm 3.2(7-20)$ & 0.7521 & $12.3 \pm 2.7(7-19)$ & 0.0340 & 0.0007 \\
\hline Group SB & $14.4 \pm 3.4(7-19)$ & & $10.7 \pm 3.6(4-19)$ & & $<0.0001$ \\
\hline
\end{tabular}

There were no significant differences in ROM between the two groups, either before surgery or at final follow-up

tendon-bone interface. In an experiment using fresh-frozen cadavers, Park et al. found that the contact area to footprint of a rotator cuff repaired by the SB method is approximately double that achieved by the DR method. They also reported that the contact pressure achieved by the SB method is $30 \%$ higher than that achieved by the DR method [8]. Additionally, Park et al. and Hatta et al. have reported that the SB method is superior to the DR method in terms of breaking strength, again according to experiments using fresh-frozen cadavers [9, 10]. Also in experiments using fresh-frozen cadavers, Steve et al. demonstrated that initial fixation strength is equivalent in that achieved by the SB and conventional trans-osseous methods. [11] Wenyong et al. found the SB method to be superior to the DR method regarding breaking strength of rotator cuffs in rabbits eight weeks after repair [12].

Consistent with these findings from basic research, many authors have reported good clinical outcomes for the SB method [13-16]. Several studies have compared the outcomes of the SB method with those of other methods of ARCR. Gary et al. analyzed ARCR for complete tears of a solitary supraspinatus tendon by the SR and SB methods and found the re-tear rate was significantly lower for the SB (7\%) than the SR method (25\%) [17]. Ide et al. analyzed clinical outcomes of ARCR using the SR and SB methods for anterosuperior rotator cuff tears and reported no significant difference in UCLA or JOA scores between the two methods. However, the re-tear rate was significantly lower for the SB (14\%) than the SR method (32\%). They also showed that functional tests of the subscapularis muscle such as the lift-off and belly press tests were performed better in the SB than the SR group [6].

Few studies have directly compared clinical outcomes between the SB and DR methods. Kim et al. compared clinical results between the DR and SB methods after
ARCR for complete rotator cuff tears of anteroposterior diameter 1-4 cm in 42 shoulders. They showed that both methods achieved significantly improved average UCLA, ASES, and Constant scores; however, they found no significant differences between the two methods. In particular, the re-tear rate did not differ significantly between the DR (24\%) and SB groups (20\%). They concluded that the SB and DR methods are comparable with regard to patient satisfaction, clinical results, and re-tear rates [18]. Recent meta-analyses have reported that the re-tear rates of the DR and SB methods are equivalent $[19,20]$. In agreement with those findings, we found no significant differences in clinical outcome, active ROM, or re-tear rate between the $\mathrm{DD}$ and $\mathrm{SB}$ methods, except internal rotation at final follow-up. It is not clear why internal rotation was better in the SB than the DD group. Kim et al. randomized patients to repair of delaminated rotator cuff tears by the DD or SB method and reported identifying no significant differences between the two groups in functional scores or ROM. However, pain scores were significantly lower in the DR than the SB group, whereas in our study the pain element of the JOA scores did not differ significantly between these groups. We believe that these apparent discrepancies are attributable to the selection criteria for the two methods. Kim et al. randomized their repair methods; thus, tears that were suitable for the DD method may have been repaired by the SB method [21].

There is an ongoing challenge to reduce re-tear rates after ARCR by employing different approaches. Healing of the repaired cuff affects re-tear rates. In this regard, Cho et al. have reported that cuffs repaired by SB have high re-tear rates $(28.9 \%)$ and suggested the possibility of necrosis of the repaired tendon [1]. Recently published basic research on RCT in rabbits has shown impaired healing in the repaired cuff after the SB method, supporting this hypothesis [22]. In their histological 
study, more vessels and less fibrinoid deposition were observed in a parallel trans-osseous repair (PTR) group than in an SB group. Biomechanical strength did not differ at 1 week; however, it was significantly worse in the SB than the PTR group at 2 weeks. In the SB group, failure in the medial row occurred with significant frequency at 2 weeks. Therefore, although SB strongly compresses the tendon to the footprint of the rotator cuff, it may negatively affect re-vascularization or fibrosis of the cuff, resulting in impairment of healing. These findings also explain the mechanism of failure around the medial anchor after the SB method, which is similar to the failure after the DR method. We and others ascertain the directions of movement of layers of delaminated cuffs to determine whether to utilize the DD or SB method. Sugaya et al. precisely analyzed the pattern of delamination of RCTs and utilized the DD or SB method depending on the direction of retraction of the delaminated cuff [2]. They achieved a $7.6 \%$ re-tear rate in the two groups combined. In our study, in which we allocated delaminated cuffs on the basis of the direction of retraction (Fig. 1), we observed low re-tear rates for both the SB (7.9\%) and DD methods (5.9\%).

Our strategy for ARCR is to choose the optimal repair technique on the basis of the type of RCT. For instance, in the DR group the tears were mostly U- or L-shaped, which cannot be optimally repaired by the SB method. However, some authors have reported converting these types of tears to a crescent-shape as a first step and then repaired the resultant crescent tear by the SB method $[23,24]$. As discussed above, the SB method is not always the best for ARCR because of both biomechanical strength and biological healing of the tendon. It is important to repair RCT by a method that does not produce excessive tension, this being achieved by careful assessment of the shape and delamination status of the torn rotator cuff.

Our study has some limitations. First, it was a small study, making it difficult to draw definite conclusions or prove our hypothesis. Second, the duration of follow-up was short. Third, the operator selected the operative method according to the condition of the torn cuffs. Finally, because we did not incorporate a control group without preoperative selection, we cannot draw the conclusion that patient selection according to the shape of the cuff tear can improve clinical outcome and decrease re-tear rate. Future studies with an appropriate control group may provide more stringent evidence concerning our hypothesis.

This study also had some strengths. First, all ARCR surgery was performed by a single surgeon. Second, to the best of our belief we are the first to report clinical results and re-tear rates for the SB and DD methods after allocating patients according to the preoperative condition of the ruptured cuff.

\section{Conclusions}

Both DD and SB methods achieve satisfactory clinical outcomes that do not differ significantly. Our results suggest that careful selection of operative method on the basis of the delamination pattern in patients undergoing $\mathrm{RCT}$ may reduce the re-tear rate after utilizing the SB method.

\section{Abbreviations \\ ARCR: Arthroscopic rotator cuff repair; DD: Double-layer, double-row; DR: Double- row; JOA score: Japanese orthopedic association score; ROM: Range of motion; SB: Suture-bridge; SR: Single-row}

\section{Acknowledgements}

We thank Dr. Trish Reynolds, MBBS, FRACP, from Edanz Group (www.edanzediting.com/ac) for editing a draft of this manuscript.

\section{Funding}

No financial support was obtained specifically for this study.

\section{Availability of data and materials}

The datasets used and/or analyzed during the current study are available from the corresponding author on reasonable request.

\section{Authors' contributions}

HK, YF and Tl participated in performing the procedures and following up the patients. TS was in charge of the statistical analysis. HK and SN wrote the manuscript. YI and SK proof read the manuscript. All authors have read and approved the final manuscript.

Ethics approval and consent to participate

This study was approved by the Ethics Committee of Kagoshima University (reference number 25-80).

Consent for publication

Consent for publication was obtained from all patients included in this study.

\section{Competing interests}

The authors declare that they have no competing interests.

\section{Publisher's Note}

Springer Nature remains neutral with regard to jurisdictional claims in published maps and institutional affiliations.

\section{Author details}

'Department of Orthopedic Surgery, Graduate School of Medical and Dental Sciences, Kagoshima University, 8-35-1 Sakuragaoka, Kagoshima City 890-8520, Japan. ${ }^{2}$ Health Service Center, National Institute of Fitness and Sports in Kanoya, 1 Shiromizu-cho, Kanoya, Kagoshima 891-2393, Japan. ${ }^{3}$ Near-Future Locomotor Organ Medicine Creation Course (Kusunoki Kai), Graduate School of Medical and Dental Sciences, Kagoshima University, 8-35-1 Sakuragaoka, Kagoshima City 890-8520, Japan. ${ }^{4}$ Department of Medical Joint Materials, Graduate School of Medical and Dental Sciences, Kagoshima University, 8-35-1 Sakuragaoka, Kagoshima City 890-8520, Japan.

Received: 9 January 2018 Accepted: 24 August 2018

Published online: 11 September 2018

\section{References}

1. Cho NS, Lee BG, Rhee YG. Arthroscopic rotator cuff repair using a suture bridge technique: is the repair integrity actually maintained? Am J Sports Med. 2011;39(10):2108-16.

2. Cha SW, Lee CK, Sugaya H, Kim T, Lee SC. Retraction pattern of delaminated rotator cuff tears: dual-layer rotator cuff repair. J Orthop Surg Res. 2016; 11(1):75.

3. Sugaya H, Maeda K, Matsuki K, Moriishi J. Repair integrity and functional outcome after arthroscopic double-row rotator cuff repair. A prospective outcome study. J Bone Joint Surg Am. 2007;89(5):953-60. 
4. DeOrio JK, Cofield RH. Results of a second attempt at surgical repair of a failed initial rotator-cuff repair. J Bone Joint Surg Am. 1984;66(4):563-7.

5. Ide J, Takagi K. Early and long-term results of arthroscopic treatment for shoulder stiffness. J Shoulder Elb Surg. 2004;13(2):174-9.

6. Ide J, Karasugi T, Okamoto N, Taniwaki T, Oka K, Mizuta H. Functional and structural comparisons of the arthroscopic knotless double-row suture bridge and single-row repair for anterosuperior rotator cuff tears. J Shoulder Elb Surg. 2015;24(10):1544-54.

7. Sugaya H, Maeda K, Matsuki K, Moriishi J. Functional and structural outcome after arthroscopic full-thickness rotator cuff repair: single-row versus dualrow fixation. Arthroscopy : the journal of arthroscopic \& related surgery official publication of the Arthroscopy Association of North America and the International Arthroscopy Association. 2005;21(11):1307-16.

8. Park MC, ElAttrache NS, Tibone JE, Ahmad CS, Jun BJ, Lee TQ. Part I: footprint contact characteristics for a transosseous-equivalent rotator cuff repair technique compared with a double-row repair technique. J Shoulder Elb Surg. 2007;16(4):461-8.

9. Park MC, Tibone JE, ElAttrache NS, Ahmad CS, Jun BJ, Lee TQ. Part II: biomechanical assessment for a footprint-restoring transosseous-equivalent rotator cuff repair technique compared with a double-row repair technique. J Shoulder Elb Surg. 2007;16(4):469-76.

10. Hatta T, Giambini H, Hooke AW, Zhao C, Sperling JW, Steinmann SP, Yamamoto N, Itoi $\mathrm{E}, \mathrm{An} \mathrm{KN}$. Comparison of passive stiffness changes in the supraspinatus muscle after double-row and knotless Transosseousequivalent rotator cuff repair techniques: a cadaveric study. Arthroscopy : the journal of arthroscopic \& related surgery : official publication of the Arthroscopy Association of North America and the International Arthroscopy Association. 2016;32(10):1973-81.

11. Behrens SB, Bruce B, Zonno AJ, Paller D, Green A. Initial fixation strength of transosseous-equivalent suture bridge rotator cuff repair is comparable with transosseous repair. Am J Sports Med. 2012;40(1):133-40.

12. Fei W, Guo W. A biomechanical and histological comparison of the suture bridge and conventional double-row techniques of the repair of full-thickness rotator cuff tears in a rabbit model. BMC Musculoskelet Disord. 2015;16:148.

13. Neyton L, Godeneche A, Nove-Josserand L, Carrillon Y, Clechet J, Hardy MB. Arthroscopic suture-bridge repair for small to medium size supraspinatus tear: healing rate and retear pattern. Arthroscopy : the journal of arthroscopic \& related surgery : official publication of the Arthroscopy Association of North America and the International Arthroscopy Association. 2013;29(1):10-7.

14. Choi S, Kim MK, Kim GM, Roh YH, Hwang IK, Kang H. Factors associated with clinical and structural outcomes after arthroscopic rotator cuff repair with a suture bridge technique in medium, large, and massive tears. J Shoulder Elb Surg. 2014;23(11):1675-81.

15. Park JY, Lhee SH, Oh KS, Moon SG, Hwang JT. Clinical and ultrasonographic outcomes of arthroscopic suture bridge repair for massive rotator cuff tear. Arthroscopy : the journal of arthroscopic \& related surgery : official publication of the Arthroscopy Association of North America and the International Arthroscopy Association. 2013;29(2):280-9.

16. Kim JR, Cho YS, Ryu KJ, Kim JH. Clinical and radiographic outcomes after arthroscopic repair of massive rotator cuff tears using a suture bridge technique: assessment of repair integrity on magnetic resonance imaging. Am J Sports Med. 2012;40(4):786-93.

17. Gartsman GM, Drake G, Edwards TB, Elkousy HA, Hammerman SM, O'Connor $D P$, Press CM. Ultrasound evaluation of arthroscopic full-thickness supraspinatus rotator cuff repair: single-row versus double-row suture bridge (transosseous equivalent) fixation. Results of a prospective, randomized study. J Shoulder Elb Surg. 2013:22(11):1480-7.

18. Kim KC, Shin HD, Lee WY, Han SC. Repair integrity and functional outcome after arthroscopic rotator cuff repair: double-row versus suture-bridge technique. Am J Sports Med. 2012:40(2):294-9.

19. Brown MJ, Pula DA, Kluczynski MA, Mashtare T, Bisson LJ. Does suture technique affect re-rupture in arthroscopic rotator cuff repair? A metaanalysis. Arthroscopy : the journal of arthroscopic \& related surgery : official publication of the Arthroscopy Association of North America and the International Arthroscopy Association. 2015;31(8):1576-82.

20. Hein J, Reilly JM, Chae J, Maerz T, Anderson K. Retear rates after arthroscopic single-row, double-row, and suture bridge rotator cuff repair at a minimum of 1 year of imaging follow-up: a systematic review. Arthroscopy : the journal of arthroscopic \& related surgery : official publication of the Arthroscopy Association of North America and the International Arthroscopy Association. 2015;31(11):2274-81.
21. Kim YS, Lee HJ, Jin HK, Kim SE, Lee JW. Conventional En masse repair versus separate double-layer double-row repair for the treatment of delaminated rotator cuff tears. Am J Sports Med. 2016;44(5):1146-52.

22. Kim SH, Kim J, Choi YE, Lee HR. Healing disturbance with suture bridge configuration repair in rabbit rotator cuff tear. J Shoulder Elb Surg. 2016; 25(3):478-86.

23. Nho SJ, Ghodadra N, Provencher MT, Reiff S, Romeo AA. Anatomic reduction and next-generation fixation constructs for arthroscopic repair of crescent, L-shaped, and U-shaped rotator cuff tears. Arthroscopy : the journal of arthroscopic \& related surgery : official publication of the Arthroscopy Association of North America and the International Arthroscopy Association. 2009;25(5):553-9.

24. Park JY, Jung SW, Jeon SH, Cho HW, Choi JH, Oh KS. Arthroscopic repair of large U-shaped rotator cuff tears without margin convergence versus repair of crescent- or L-shaped tears. Am J Sports Med. 2014;42(1):103-11.
Ready to submit your research? Choose BMC and benefit from:

- fast, convenient online submission

- thorough peer review by experienced researchers in your field

- rapid publication on acceptance

- support for research data, including large and complex data types

- gold Open Access which fosters wider collaboration and increased citations

- maximum visibility for your research: over $100 \mathrm{M}$ website views per year

At BMC, research is always in progress.

Learn more biomedcentral.com/submissions 